\title{
A retrospective analysis of the accuracy of radioactively labeled autologous leukocytes in patients with infected prosthetic joints
}

Lindsay Brammen¹, Christopher J. Palestro², Johannes Holinka ${ }^{3}$, Reinhard Windhager ${ }^{3}$, Helmut Sinzinger ${ }^{4,5}$

${ }^{1}$ Department of Surgery, Division of General Surgery, Medical University of Vienna, Austria

2Division of Nuclear Medicine \& Molecular Imaging, Long Island Jewish Medical Center, New Hyde Park, USA

${ }^{3}$ Department of Orthopedic Surgery, Medical University of Vienna, Austria

${ }^{4}$ ISOTOPIX — Institute for Nuclear Medicine, Vienna, Austria

${ }^{5}$ Department of Nuclear Medicine, Medical University of Vienna, Austria

[Received 25 XII 2016; Accepted 4 IV 2017]

\begin{abstract}
BACKGROUND: Labeled leukocyte scintigraphy (LS) is considered a valuable tool in preoperative diagnosis of prosthetic joint infections (PJI). The aim of this study was to determine the accuracy of LS combined with bone marrow scintigraphy (BMS), as well as inflammation markers CRP and WBC, in detecting infection in patients with prosthetic joints.

MATERIAL AND METHODS: This study included patients suspected of having PJI between January and September 2013 at the Vienna General Hospital who underwent imaging with ${ }^{99 m}$ Tc-HMPAO labeled autologous leukocytes and subsequent BMS. Diagnostic accuracy was assessed in terms of sensitivity, specificity, positive predictive value (PPV) and negative predictive value (NPV).

RESULTS: A total of 48 patients were included. The most common joint investigated was knee (25), followed by hip (9), shoulder (2), and elbow (1). Other parts of the body investigated included the femur (6), tibia (2), leg (2), and foot (1). The pathogens most frequently isolated included Staphylococcus epidermidis and Candida albicans. The sensitivity of LS was $60 \%$, specificity 97\%, PPV $86 \%$ and NPV $90 \%$. Overall accuracy was calculated to be $90 \%$.

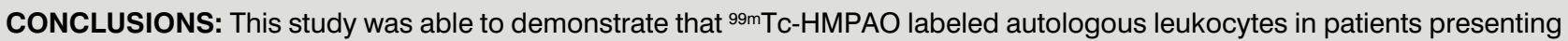
with symptoms of PJI is accurate. In contrast, however, inflammation markers CRP and WBC are not accurate pre-diagnostic markers for PJI.
\end{abstract}

KEY words: leukocyte labeling, prosthetic joint, infection, ${ }^{99 m T C-H M P A O, ~ s c i n t i g r a p h y ~}$

Nucl Med Rev 2017; 20, 2: 81-87

\section{Background}

Given that life expectancy is gradually increasing, prosthetic joint replacement is more frequently being utilized as a way of improving the quality of life in an ever-ageing population [1]. Nevertheless, there are risks and complications, as with every surgical proce-

Correspondence to: Helmut Sinzinger MD, Prof.

ISOTOPIX, Institute of Nuclear Medicine

Mariannengasse 30, A-1090 Vienna, Austria

Tel.: + 43 (0)1 4082633

Fax: $+43(0) 14081366$

E-mail: helmut.sinzinger@meduniwien.ac.at dure. Common complications seen after joint replacement surgery include aseptic or mechanical loosening, as well as polyethylene wear [2]. While not frequently observed, serious complications, such as prosthetic joint infection, can result in significant morbidity, prolonged invalidity and hospitalization, as well as decrease in joint function, all of which often lead to explantation and subsequent re-implantation subsequent to several weeks of antibiotic therapy $[1,3]$. Following primary hip implantation, there is a $1 \%$ rate of infection, while a $2 \%$ rate of infection has been reported in knee prostheses [4]. After revision surgery, these percentages increase to about $3 \%$ for hip replacements and $5 \%$ for knee replacements [4].

Prosthetic joint infections (PJI) can be classified into "early" (within three months after surgery), "delayed" (between three 
months to two years) and "late" (after two years) [5]. The two most common organisms in PJI include Staphylococcus epidermidis (31\%) and Staphylococcus aureus (20\%) [2]. While Staphylococcus aureus is typically seen in "early" joint infections, coagulase-negative Staphylococci, Streptococci, Enterococci and anaerobes are observed in "late" infections [5]. Several factors that predispose individuals to PJI are obesity, poor nutritional status, diabetes mellitus, higher age, remote infection, immune suppression, underlying joint inflammation (such as rheumatoid arthritis, psoriasis), and prior joint infection [6, 7].

PJI is defined by major and minor criteria by the Musculoskeletal Infection Society (MSIS). Major criteria include: a) presence of a sinus tract communicating with the prosthesis or b) two positive periprosthetic cultures with phenotypically identical organisms [8]. Minor criteria include: 1) raised serum erythrocyte sedimentation rate (ESR) and serum C-reactive protein concentration (CRP), 2) raised synovial WBC count change on leucocyte esterase test strip, 3) raised synovial polymorphonuclear neutrophil percentage, 4) positive histological analysis of periprosthetic tissue or 5) a single positive culture [8]. Tenacious pain after surgery or a persistent marginally elevated CRP result in difficult diagnosis situations [1]. Therefore, diagnosis involves a variety of different factors. Firstly, thorough clinical histories, including medical, surgical and physical examinations deliver excellent initial diagnostic and aid in subsequent diagnostic evaluation [1]. Further diagnostic evaluation of PJI includes a physical examination, hematological tests including inflammation markers [C-reactive protein (CRP), white blood cell (WBC) count and erythrocyte sedimentation rate (ESR), interleukin-6], joint aspiration and numerous imaging modalities [1, 9]. A normal CRP or ESR cannot completely rule out a low-grade infection, given that false negative results can occur following long-term antibiotic treatment or in patients with delayed-onset infection [9]. Therefore, additional diagnostic examinations, such as joint aspiration with a WBC count and differential, gram stain and culture, as well as numerous imaging modalities may be required [1, 9]. Unfortunately, the MSIS criteria lacks a standardized diagnostic definition, which could improve diagnostic accuracy in studies on PJI [10].

It is well known that bacteria secrete chemotactic factors, such as prostaglandins and histamine. The secretion of these factors results in the recruitment of leukocytes, induction of endothelial activation and edema. Hence, the persistent recruitment of leukocytes from the blood to the periprosthetic tissue is characteristic of an acute or sub-acute bacterial infection. Autologous radiolabelled white blood cells have a high specificity given that they actively migrate into an infected tissue via adherence to vascular endothelium [11]. In the infected joint, neutrophils are the predominant labeled circulating cells in labeled leukocyte scintigraphy (LS) using tracers such as ${ }^{111}$ In-oxine and ${ }^{99 m}$ Tc-hexamethyl propyleneamine oxime (HMPAO) [1] . Leukocyte labeling was first introduced in 1976 by McAfee and Thakur [12]. In the absence of infection or where there is no increased bone turnover, there is no accumulation of labeled leukocytes [1]. Therefore, LS is deemed a valuable tool in the diagnosis of PJI [1]. Since neutrophils are typically absent in aseptic loosened prosthesis, LS should be able to differentiate between an inflamed aseptic prosthesis and an infected prosthesis [4]. However, one must take into consideration that bone marrow displacement or activation through surgery can give rise to a secondary uptake of leukocytes around the prosthesis [1]. Consequently, a combination of LS with BMS using ${ }^{99 m}$ Tc-sulphur-/nanocolloid has been introduced [1] . The uptake of sulfur-/nano colloid within 48 hours of bacterial seeding is suppressed by low oxygen tension, increased intraosseous pressure, acidic $\mathrm{pH}$ and vascular insufficiency [13]. Since infection, on the one hand, stimulates the uptake of leukocytes, but on the other hand, suppresses the uptake of sulfur-/nanocolloid, LS and BMS are spatially incongruent in infection [4]. If the uptake of the two radiopharmaceuticals is similar or spatially congruent, the labeled leukocyte activity is attributable to bone marrow uptake [4]. The combination of LS and BMS in detecting infection has a reported accuracy of 86-98\% [14-25]. LS has also been employed in patients with systemic infections, postoperative infections and in fever of unknown origin [26]. The indications for a combination imaging LS/BMS include prosthetic joint infection, musculoskeletal infections, and neuropathic joint [26].

The aim of this study was to examine imaging of LS combined with BMS in their accuracy and reliability in detecting infection in patients with prosthetic joints. Furthermore, inflammation markers CRP and WBC count were analyzed for their accuracy in detecting PJI.

\section{Material and methods}

\section{Patients}

This study included a total of 48 patients ( 23 male, 25 female) suspected of having PJI diagnosed according to MSIS criteria by orthopedic surgeons between January and September 2013 at the Vienna General Hospital. In order to be diagnosed with PJI and included into the study, the patients had to fulfill one major criterion or three minor MSIS criteria. Each patient suffered from symptoms such as pain and swelling. Inflammation markers were analyzed in the blood in each patient one to two days before undergoing LS/BMS. All patients underwent antibiotic therapy for at least four weeks prior to LS/BMS. Informed consent was obtained from all individual participants included in the study, making them aware of the procedure they were undergoing, the risks and that they released their data to be used in clinical studies. The study protocol was approved by the local ethical committee (EK-reference number 1948/2013) and the study was carried out according to the provisions of the Declaration of Helsinki.

\section{Leukocyte labeling}

In each patient, approximately four $20 \mathrm{~mL}$ blood vials, each anticoagulated with $2 \mathrm{~mL}$ acid citrate dextrose (ACD), was filled with blood drawn from a peripheral vein. The leukocytes were separated and radioactively labeled according to protocol (10 min, $22^{\circ} \mathrm{C}$ ) using Leuco-scint-kits (Medi-Radiopharma, Hungary). The labeled leukocytes were then re-suspended and re-injected into a peripheral vein with a delay of no more than 150 minutes [27]. The mean administered activity of ${ }^{99 m} \mathrm{Tc}-\mathrm{HMPAO}$ was approximately $700 \mathrm{MBq}$. Labeling efficiency ranged between 61 and 85\%.

\section{Imaging procedures}

After leukocyte labeling with ${ }^{99 m} \mathrm{Tc}-\mathrm{HMPAO}$, patients underwent whole body scintigraphy and local images in both anterior and posterior views and if required, lateral views, were recorded with a double-headed gamma camera four hours after re-injection. Additional local images in the necessary views were taken ap- 
proximately 24 hours following re-injection. In the case of positive LS, bone marrow scintigraphy was conducted 48 hours after re-injection. Local images were recorded 30 minutes after injection of $370 \mathrm{MBq}{ }^{99 m} \mathrm{Tc}-$ nanocolloid (GE Healthcare AG, Switzerland). The same gamma camera was used for all acquisitions. The scans were visually analyzed and considered positive when leukocyte uptake intensity in the region of interest was present, yet there was no corresponding bone marrow uptake in the same region. The scans were considered negative when bone marrow uptake in the region of interest corresponded to leucocyte uptake intensity.

\section{Statistical analysis}

Descriptive statistics included the following parameters: age, gender, diagnosis, labeling efficiency, infection parameters in blood, intraoperative bacterial culture result, intraoperative histological culture result and end clinical result. Accuracy was determined in terms of sensitivity, specificity, positive predictive value and negative predictive value.

P-values $<0.05$ were considered statistically significant. Analysis of data was performed using SPSS (Statistical Package for Social Sciences, Chicago, IL, USA) v19 for Windows.

\section{Results}

During the duration of the study period from January to September 2013, 48 patients suspected of having prosthetic joint infections underwent ${ }^{99 m} \mathrm{Tc}-\mathrm{HMPAO}$ labeled autologous leukocyte imaging. All patients underwent subsequent bacterial and histological testing via joint aspiration or operation. Patients' characteristics are provided in Table 1.

There were ten cases of infection. The most common pathogens isolated from the joints included Staphylococcus epidermidis and Candida albicans. Bacterial testing diagnosed S. epidermidis infections in five patients and $C$. albicans in one patient.

Six out of our 48 patients, were determined to be true positive, 37 true negative, one false positive and four false negative. Images are provided in Figure 1 and 2. In order to assess the accuracy of this technique, we calculated sensitivity, specificity, positive predictive value and negative predictive value. The calculated sensitivity of LS was $60 \%$, specificity $97 \%$, PPV $86 \%$ and NPV $90 \%$. Therefore, overall accuracy was $90 \%$.

\section{Table 1. Patients characteristics}

$\begin{array}{lc}\text { Number of patients } & 48 \\ \text { Males:Females } & 25: 23 \\ \text { Mean age (years) } & 60 \text { (range 21-84) } \\ \text { Affected joints (numbers) } & 25 \\ \quad \text { Knee } & 9 \\ \text { Hip } & 2 \\ \quad \text { Shoulder } & 1 \\ \quad \text { Elbow } & 9 \\ \quad \text { Other } & 3.9(0.05-13.4) \\ \text { Mean CRP [mg/dl] (range) } & 4.8(0.09-11.1) \\ \text { Mean leukocyte count [G/l] (range) } & 61-85 \\ \text { Labeling efficiency range (\%) }\end{array}$

After analysis of the patients' charts, pre-diagnostic testing with CRP and WBC was conducted in 43 patients. Mean CRP was calculated to be $3,9 \mathrm{mg} / \mathrm{dL}$ (cut-off $0,5 \mathrm{mg} / \mathrm{dL}$ ). Thirty-one of our patients had a CRP-level higher than the cut-off, with 12 patients having a CRP below the cut-off. The sensitivity was calculated to be $57 \%$, specificity $28 \%$, PPV $13 \%$, NPV $77 \%$ and accuracy $33 \%$. On the other hand, three patients had WBC counts higher than the cut-off, while the remaining had WBC counts lower than the cut-off. WBC count had a sensitivity of $0 \%$, specificity $92 \%$, PPV $0 \%$, NPV $88 \%$ and overall accuracy $82 \%$.

\section{Discussion}

The reported accuracy of LS/BMS ranges from $86-98 \%$ of patients [14-25]. One of the earliest studies with 30 patients examining labeled leukocytes with bone marrow imaging and hip arthroplasty by Mulamba et al. [28] observed a 92\% sensitivity and $100 \%$ specificity for diagnosing hip infections. Another study of LS/BMS in 72 patients with hip arthroplasty demonstrated 100\% sensitivity and $97 \%$ specificity in diagnosing infection [15]. A review examining 59 patients with failed hip- and knee arthroplasties by Love at al. [24] reported the sensitivity, specificity and accuracy of combined LS/BMS to be $100 \%, 91 \%$, and $95 \%$, respectively. In addition, a study by El Espera et al. [23] determined $80 \%$ sensitivity, $94 \%$ specificity and $91 \%$ accuracy in 60 patients with knee or hip arthroplasty that received combined LS/BMS. While most studies show that combined LS/BMS is highly specific, the sensitivity of this method can vary. A study by Pill et al. [29] reported only $50 \%$ sensitivity in combined LS/BMS. Furthermore, while Joseph et al. [21] reported $100 \%$ specificity in their patient population of 58 patients with total knee or hip arthroplasty, they observed only $46 \%$ sensitivity for combined LS/BMS. In this study, we observed a low sensitivity of $60 \%$. While our sensitivity was low compared to most studies, it was higher than a study by Joseph et al. [21]. This low sensitivity rate was due to the number of false negative results. However, after reviewing the leukocyte scans with one of the leading nuclear medicine specialists in this field, our false negative rate became $0 \%$. Therefore, our sensitivity would therefore be $100 \%$. There are a few reasons as so why the patients were reported as negative in their leukocyte scans. Firstly, after review of the patient's records, it was determined that all patients had received antibiotic therapy (one patient Levofloxacin, two patients Cephalexin, one patient Clindamycin) for at least four weeks prior to their leukocyte scans. Antibiotic therapy can result in clearing of the acute infection, thus making it difficult to determine an infection in a leukocyte scan. However, after review of all of the patients' records, it was determined that a total of 24 patients (six true positive, four false negative, 14 true negative) were under antibiotic therapy when they received their leukocyte scans. Therefore, antibiotic therapy alone could not be responsible for a false negative report, in accordance to other studies [30]. Furthermore, when reporting on LS there is a learning curve, which must be taken into consideration when starting this procedure in a hospital. In our department, LS had not been routinely conducted until we began again in January 2013. In our study, we observed a high specificity of $97 \%$, which is in accordance with other studies, despite our small patient population. Our overall accuracy 
a)

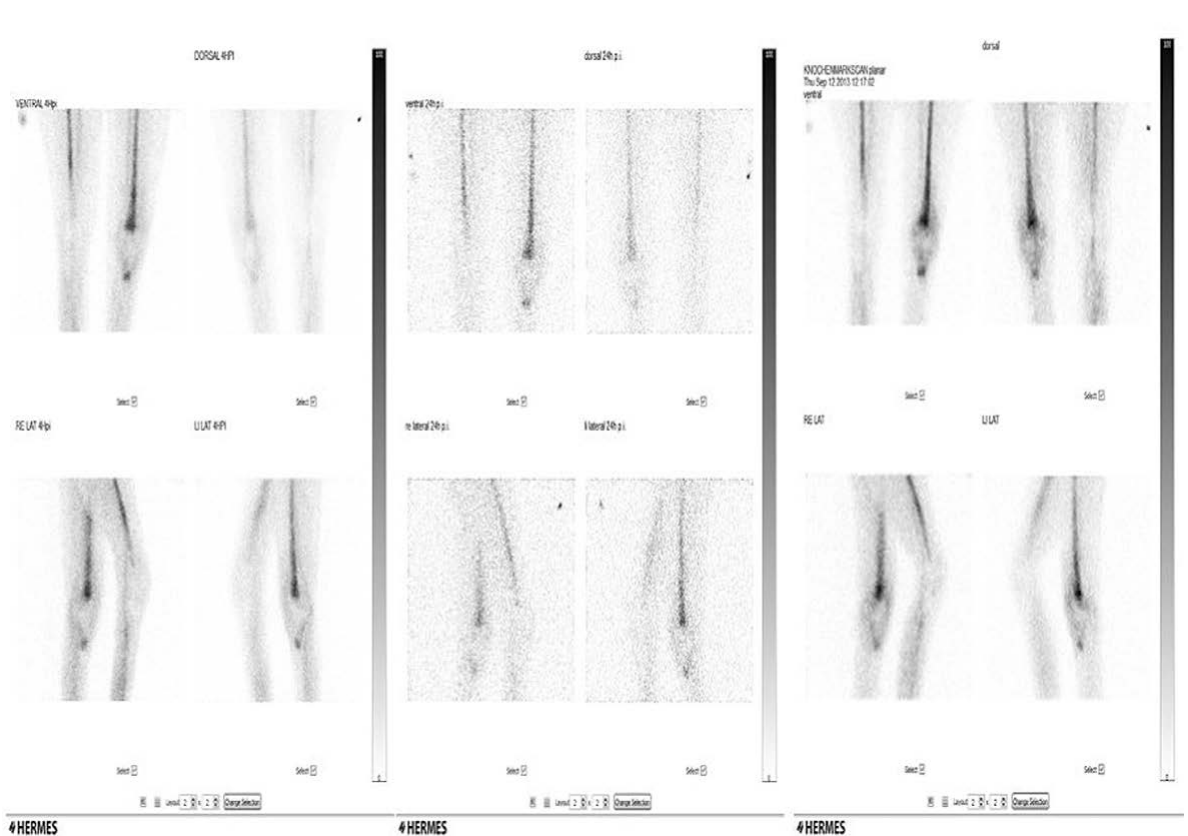

c)

Figure 1. True negative LS: A. WBC after 4h; B. WBC after 24 hours; C. bone marrow

a)
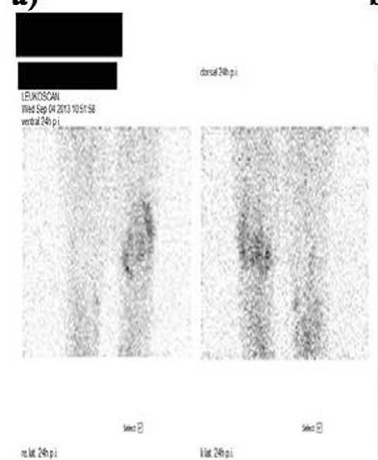

tatsi
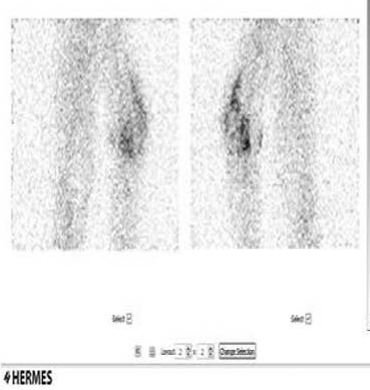

b)

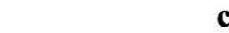

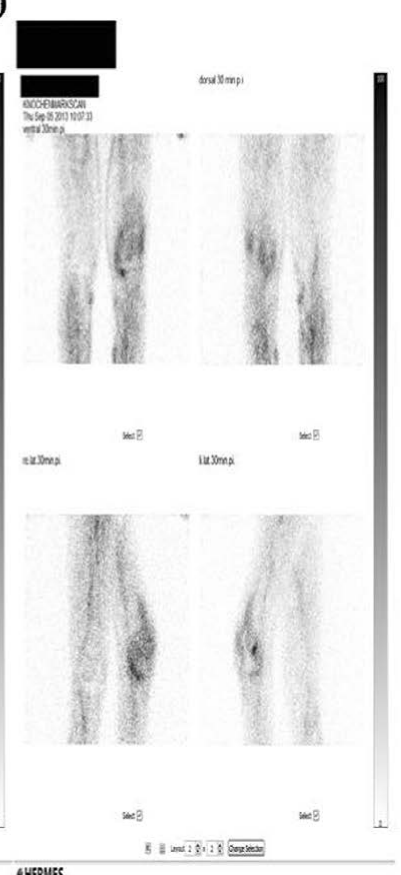

Figure 2. True positive LS: A. WBC after 4h; B. WBC after 24 hours; C. bone marrow

was calculated to be $90 \%$, lying well within the reported values. The correlation of our results with histological or bacteriological results was possible in all 48 patients. Interestingly in our study, six patients only underwent aspiration. Of these six patients, four were true negative and two were true positive. The remaining 42 patients underwent subsequent surgical exploration, all of which received both histological and bacteriological testing. Therefore, the results of the patients who only received aspiration were not related to the false negative or false positive cases. While a recently published systematic review questions the role of nuclear medicine in the diagnosis of PJI and calls for comparative assessments of diagnostic performance employing the MSIS criteria as a standard reference all while using study designs that minimize bias [10], we were nonetheless able to show that LS was accurate in our small patient population in patients diagnosed with prosthetic joint infection according to the MSIS criteria. 
It has been argued that poor sensitivity can be attributed to the chronicity of an infection, as well as non-specific inflammation [31-33]. While chronic infections are typically characterized by less distinct neutrophil recruitment and edema [34], a study by Datz et al. [35] that examined the labeled leukocytes in acute and chronic infections found no significant statistical difference in sensitivity. In non-specific inflammation, neutrophils are generally absent [36]. Given that LS is most sensitive in imaging neutrophil-dominate responses [36], aseptic inflammation may lead to false negative results and a decrease in sensitivity [37]. On the other hand, one must also consider the actual interpretation of the images. An image is deemed as positive when there is higher uptake in the region of interest than a predetermined reference point [26]. Given that infection stimulates the uptake of leukocytes but suppresses the uptake of sulfur colloid in the bone marrow, a positive image will be spatially incongruent [1]. However, one must keep in consideration that both the activity and uptake can vary, as well as the normal distribution of WBC's in the bone marrow [26]. For example, one would expect to see fewer WBC's migrating to the joints of chronic infection. Furthermore, uptake depends on the number of WBC's that migrate to the site of infection [26].

Despite the high accuracy of this technique, LS has its disadvantages, which have to be considered before performing this test. First, the procedure is labor intensive. Given that two to three technologists are involved in the labeling and imaging processes, it can be estimated that a total of 8-10 hours is required from them for this technique, over two days. Furthermore, this technique is routinely available in all only a few hospitals worldwide. In addition, it involves contact with blood products, which requires strict protocols, such as the use of a laminar flow hood [22, 24, 36].

In terms of the inflammation markers WBC count and CRP, our study included 43 patients who had pre-diagnostic CRP and WBC count analyzed. These patients had a mean CRP of $3,9 \mathrm{mg} / \mathrm{dL}$ (cut-off $0,5 \mathrm{mg} / \mathrm{dL}$ ). The sensitivity was only $57 \%$ and specificity $28 \%$, with overall accuracy being $33 \%$. We believe that CRP is not an accurate pre-diagnostic marker for PJI. However, our values are in contrast to a review by Yuan et al. [38] that demonstrated that CRP had good diagnostic accuracy for periprosthetic infections with a sensitivity of $82 \%$ and specificity of $77 \%$. In addition, a study by Glithero et al. [39] examining CRP values in patients with suspected PJI reported a sensitivity, specificity and accuracy of $83 \%, 74 \%$, and $77 \%$, respectively. One reason for our low accuracy could be due to our small patient population compared to the large ones in both studies. On the other hand, the mean WBC count was $4,8 \mathrm{G} / \mathrm{l}$ (cut-off $10 \mathrm{G} / \mathrm{l}$ ). Of our patient population, only 3 had increased WBC count, while the remaining patients had all a normal WBC count. This study demonstrated a sensitivity of $0 \%$, but high specificity of $92 \%$ and overall accuracy of $82 \%$. Therefore, a normal WBC count could be highly specific for the absence of infection. However, we must also theorize that WBC count is also not an accurate blood marker for diagnosing PJI. This is in accordance to a study by Berbari et al. [9] that demonstrated that WBC count has the lowest diagnostic accuracy for PJI. While this analysis only investigated serum CRP and WBC count, a study by Claassen et al. [40] that assessed 46 patients with knee arthroplasty and aspiration in 77 cases, demonstrated an increase in WBC count in only 7 cases and normal levels in the remaining patients [40]. In addition, CRP was increased in 33 cases and normal in 44 cases [40]. Similar to our study, they also concluded that CRP and WBC are not accurate in diagnosing ongoing infection [40].

Future directions in leukocyte labeling according to recently published papers include SPECT-CT as an adjunct to scintigraphy, as well as ${ }^{18} \mathrm{~F}$-FDG-PET-(CT). Several studies have demonstrated positive results in utilizing SPECT-CT in infection diagnostics, given the improvement in localization and defining the extent of disease. Based on current knowledge, it is supposed that the CT component increases sensitivity by accurately localizing the anatomical site of infection due to the detection of morphologic abnormalities that are parallel to sites of increased activity[41]. For example, Filippi et al.

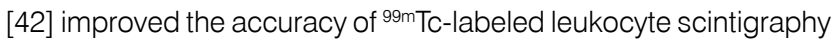
from $64 \%$ to $100 \%$. Moreover, a recently published study by Kim et al. [43], which assessed adding SPECT-CT to ${ }^{99 m}$ Tc-HMPAO-labeled leukocytes, demonstrated an increase in sensitivity from 82\% to 93.3\%, specificity $88 \%$ to $93.3 \%$, PPV from $89 \%$ to $94.3 \%$ and NPV $80.5 \%$ to $92.1 \%$ and diagnostic accuracy from $84.8 \%$ to $93.3 \%$ by correctly identifying the site of infection. Furthermore, Graute et al. [44] were able to increase sensitivity from $66 \%$ to $89 \%$ and specificity from $60 \%$ to $73 \%$ by combining planar images with SPECT-CT. However, one must also take into consideration the high radiation emitted by CT. In addition, while the CT component of SPECT-CT imaging is thought to increase sensitivity, an increase in sensitivity ultimately leads to a decrease in specificity. In terms of the role of ${ }^{18} \mathrm{~F}-\mathrm{FDG}$ PET-(CT) in prosthetic joint infection imaging, several studies have been conducted showing varying results. Gravius et al. [45] and Basu et al. [46] reported similar sensitivities of $93 \%$ and $94.7 \%$ and specificities of $83 \%$ and $88.2 \%$, respectively, in detecting infection after total knee arthroplasty. The accuracy of detecting infection in a knee prosthesis reported by Zhuang et al. [47] was quite low at $77.8 \%$. When it comes to infection following hip arthroplasty, the accuracy ranges between 89-95\% [47-49]. On the other hand, studies have shown contradictory results when it comes to ${ }^{18} \mathrm{~F}$-FDG-PET-(CT) in prosthetic joint infection. Stumpe et al. [50] and Love et al. [24] reported accuracies of only $69 \%$ and $71 \%$ in diagnosing lower joint infections with ${ }^{18} \mathrm{~F}-\mathrm{FDG}$, respectively. In addition, Garcia-Barrechguren et al. [51] demonstrated a sensitivity and specificity of $64 \%$ and $67 \%$, respectively in patients being tested for prosthetic hip infection. Moreover, Stumpe et al. [52] and Delank et al. [53] concluded in their studies that ${ }^{18} \mathrm{~F}-\mathrm{FDG}$ PET is not specific for infection. However, a recently published meta-analysis reported a pooled sensitivity and specificity of $86 \%$ in ${ }^{18} \mathrm{~F}$-FDG-PET and PET-CT examinations in patients with lower extremity prosthetic joint infection [54]. Hence, the high specificity in ${ }^{99 m} \mathrm{mc}-\mathrm{HMPAO}$ labeled autologous leukocytes is of greater importance in joint infections and therefore this technique is considered the gold standard. In conclusion, while the roles of additional SPECT-CT and ${ }^{18}$ F-FDG-PET-CT look promising in the diagnosis of prosthetic joint infection, further studies, especially comparative studies between these diagnostic modalities and LS, need to be conducted.

In conclusion, this study was able to demonstrate that scintigraphy with ${ }^{99 m} \mathrm{Tc}-\mathrm{HMPAO}$ labeled autologous leukocytes in patients presenting with symptoms of prosthetic joint infection is accurate, especially in terms of a high specificity and negative predictive value. Inflammation markers CRP and WBC count are 
not accurate in diagnosing prosthetic joint infections. In accordance with other studies, it has been shown that LS combined with BMS is a feasible imaging method in patients with infection of prosthetic joints and may also be a helpful tool in other infection scenarios.

\section{Conflict of interest and source of funding}

No conflict of interest and no financial disclosures.

\section{References}

1. Gemmel F, Van den Wyngaert $\mathrm{H}$, Love $\mathrm{C}$, et al. Prosthetic joint infections: radionuclide state-of-the-art imaging. Eur J Nucl Med Mol Imaging. 2012; 39(5): 892-909, doi: 10.1007/s00259-012-2062-7, indexed in Pubmed: 22361912.

2. Love C, Marwin SE, Palestro CJ. Nuclear medicine and the infected joint replacement. Semin Nucl Med. 2009; 39(1): 66-78, doi: 10.1053/j.semnuclmed.2008.08.007, indexed in Pubmed: 19038601.

3. Lazzeri E, Manca M, Molea N, et al. Clinical validation of the avidin/indium-111 biotin approach for imaging infection/inflammation in orthopaedic patients. Eur J Nucl Med. 1999; 26(6): 606-614, indexed in Pubmed: 10369946.

4. Love C, Tomas MB, Marwin SE, et al. Role of nuclear medicine in diagnosis of the infected joint replacement. Radiographics. 2001; 21(5): 1229-1238, doi: 10.1148/radiographics.21.5.g01se191229, indexed in Pubmed: 11553828.

5. Trampuz A, Zimmerli W, Zimmerli W, et al. Prosthetic-joint infections. N Engl J Med. 2004; 351(16): 1645-1654, doi: 10.1056/NEJMra040181, indexed in Pubmed: 15483283.

6. Zimmerli W. Infection and musculoskeletal conditions: Prosthetic-joint-associated infections. Best Pract Res Clin Rheumatol. 2006; 20(6): 1045-1063, doi: 10.1016/j.berh.2006.08.003, indexed in Pubmed: 17127196.

7. Cataldo MA, Petrosillo N, Cipriani M, et al. Prosthetic joint infection: recent developments in diagnosis and management. J Infect. 2010; 61(6): 443-448, doi: 10.1016/j.jinf.2010.09.033, indexed in Pubmed: 20932998.

8. Parvizi J, Gehrke T, Chen AF. Proceedings of the International Consensus on Periprosthetic Joint Infection. Bone Joint J. 2013; 95-B(11): 1450-1452, doi: 10.1302/0301-620X.95B11.33135, indexed in Pubmed: 24151261

9. Berbari E, Mabry T, Tsaras G, et al. Inflammatory blood laboratory levels as markers of prosthetic joint infection: a systematic review and meta-analysis. J Bone Joint Surg Am. 2010; 92(11): 2102-2109, doi: 10.2106/JBJS.I.01199, indexed in Pubmed: 20810860.

10. Diaz-Ledezma C, Lamberton C, Lichstein P, et al. Diagnosis of Periprosthetic Joint Infection: The Role of Nuclear Medicine May Be Overestimated. J Arthroplasty. 2015; 30(6): 1044-1049, doi: 10.1016/j.arth.2015.01.008, indexed in Pubmed: 25686785

11. Datz FL. Indium-111-labeled leukocytes for the detection of infection: current status. Semin Nucl Med. 1994; 24(2): 92-109, indexed in Pubmed: 8023176.

12. McAfee JG, Thakur ML. Survey of radioactive agents for in vitro labeling of phagocytic leukocytes. II. Particles. J Nucl Med. 1976; 17(6): 488-492, indexed in Pubmed: 1262966

13. Mader JT, Brown GL, Guckian JC, et al. A mechanism for the amelioration by hyperbaric oxygen of experimental staphylococcal osteomyelitis in rabbits. J Infect Dis. 1980; 142(6): 915-922, indexed in Pubmed: 7462700.

14. King AD, Peters AM, Stuttle AW, et al. Imaging of bone infection with labelled white blood cells: role of contemporaneous bone marrow imaging. Eur J Nucl Med. 1990; 17(3-4): 148-151, indexed in Pubmed: 2279496.

15. Palestro CJ, Kim CK, Swyer AJ, et al. Total-hip arthroplasty: periprosthetic indium-111-labeled leukocyte activity and complementary technetium-99m-sulfur colloid imaging in suspected infection. J Nucl Med. 1990; 31(12): 1950-1955, indexed in Pubmed: 2266391.

16. Palestro CJ, Swyer AJ, Kim CK, et al. Infected knee prosthesis: diagnosis with In-111 leukocyte, Tc-99m sulfur colloid, and Tc-99m MDP imaging. Radiology. 1991; 179(3): 645-648, doi: 10.1148/radiology.179.3.2027967, indexed in Pubmed: 2027967
17. Seabold JE, Nepola JV, Marsh JL, et al. Postoperative bone marrow alterations: potential pitfalls in the diagnosis of osteomyelitis with In-111-labeled leukocyte scintigraphy. Radiology. 1991; 180(3): 741-747, doi: 10.1148/radiology.180.3.1871288, indexed in Pubmed: 1871288.

18. Palestro CJ, Roumanas P, Swyer AJ, et al. Diagnosis of musculoskeletal infection using combined In-111 labeled leukocyte and Tc-99m SC marrow imaging. Clin Nucl Med. 1992; 17(4): 269-273, indexed in Pubmed: 1572113.

19. Achong DM, Oates $\mathrm{E}$. The computer-generated bone marrow subtraction image: a valuable adjunct to combined In-111 WBC/Tc-99m in sulfur colloid scintigraphy for musculoskeletal infection. Clin Nucl Med. 1994; 19(3): 188-193, indexed in Pubmed: 8033465.

20. Palestro CJ, Mehta HH, Patel M, et al. Marrow versus infection in the Charcot joint: indium-111 leukocyte and technetium-99m sulfur colloid scintigraphy. J Nucl Med. 1998; 39(2): 346-350, indexed in Pubmed: 9476948.

21. Joseph TN, Mujtaba M, Chen AL, et al. Efficacy of combined technetium-99m sulfur colloid/indium-111 leukocyte scans to detect infected total hip and knee arthroplasties. J Arthroplasty. 2001; 16(6): 753-758, doi: 10.1054/arth.2001.24446, indexed in Pubmed: 11547374.

22. Palestro CJ. Nuclear medicine, the painful prosthetic joint, and orthopedic infection. J Nucl Med. 2003; 44(6): 927-929, indexed in Pubmed: 12791821.

23. El Espera I, Blondet C, Moullart V, et al. The usefulness of $99 \mathrm{mTc}$ sulfur colloid bone marrow scintigraphy combined with $111 \mathrm{In}$ leucocyte scintigraphy in prosthetic joint infection. Nucl Med Commun. 2004; 25(2): 171-175, indexed in Pubmed: 15154708.

24. Love C, Marwin SE, Tomas MB, et al. Diagnosing infection in the failed joint replacement: a comparison of coincidence detection 18F-FDG and 1111 In-labeled leukocyte/99mTc-sulfur colloid marrow imaging. J Nucl Med. 2004; 45(11): 1864-1871, indexed in Pubmed: 15534056.

25. Fuster D, Duch J, Soriano A, et al. [Potential use of bone marrow scintigraphy in suspected prosthetic hip infection evaluated with 99mTc-HMPAO-leukocytes]. Rev Esp Med Nucl. 2008; 27(6): 430-435, indexed in Pubmed: 19094902.

26. Palestro CJ, Love C, Tronco GG, et al. Combined labeled leukocyte and technetium 99m sulfur colloid bone marrow imaging for diagnosing musculoskeletal infection. Radiographics. 2006; 26(3): 859-870, doi: 10.1148/rg.263055139, indexed in Pubmed: 16702459.

27. Devillers A, Moisan A, Jean S, et al. Technetium-99m hexamethylpropylene amine oxime leucocyte scintigraphy for the diagnosis of bone and joint infections: a retrospective study in 116 patients. Eur J Nucl Med. 1995; 22(4): 302-307, indexed in Pubmed: 7607260

28. Mulamba L, Ferrant A, Leners N, et al. Indium-111 leucocyte scanning in the evaluation of painful hip arthroplasty. Acta Orthop Scand. 1983; 54(5): 695-697, indexed in Pubmed: 6670484.

29. Pill SG, Parvizi J, Tang PH, et al. Comparison of fluorodeoxyglucose positron emission tomography and (111)indium-white blood cell imaging in the diagnosis of periprosthetic infection of the hip. J Arthroplasty. 2006; 21(6 Suppl 2): 91-97, doi: 10.1016/j.arth.2006.05.021, indexed in Pubmed: 16950069.

30. Sinzinger $\mathrm{H}$, Granegger $\mathrm{S}$. The effect of various antibiotics on the labelling efficiency of human white blood cells with 111 In-oxine. Nucl Med Commun. 1988; 9(8): 597-601, indexed in Pubmed: 3140148.

31. Al-Sheikh W, Sfakianakis GN, Mnaymneh W, et al. Subacute and chronic bone infections: diagnosis using In-111, Ga-67 and Tc-99m MDP bone scintigraphy, and radiography. Radiology. 1985; 155(2): 501-506, doi: 10.1148/radiology.155.2.3157204, indexed in Pubmed: 3157204.

32. Johnson JA, Christie MJ, Sandler MP, et al. Detection of occult infection following total joint arthroplasty using sequential technetium-99m HDP bone scintigraphy and indium-111 WBC imaging. J Nucl Med. 1988; 29(8): 1347-1353, indexed in Pubmed: 3404252.

33. McKillop JH, McKay I, Cuthbert GF, et al. Scintigraphic evaluation of the painful prosthetic joint: a comparison of gallium-67 citrate and indium-111 labelled leucocyte imaging. Clin Radiol. 1984; 35(3): 239-241, indexed in Pubmed: 6425000 . 
34. Glaudemans AW, Galli F, Pacilio M, et al. Leukocyte and bacteria imaging in prosthetic joint infection. Eur Cell Mater. 2013; 25: 61-77, indexed in Pubmed: 23325539.

35. Datz FL, Thorne DA. Effect of chronicity of infection on the sensitivity of the In-111-labeled leukocyte scan. AJR Am J Roentgenol. 1986; 147(4): 809-812, doi: 10.2214/ajr.147.4.809, indexed in Pubmed: 3489385.

36. Palestro CJ. Nuclear medicine and the failed joint replacement: Past, present, and future. World J Radiol. 2014; 6(7): 446-458, doi: 10.4329/wjr. v6.i7.446, indexed in Pubmed: 25071885.

37. Palestro CJ, Love C, Bhargava KK. Labeled leukocyte imaging: current status and future directions. Q J Nucl Med Mol Imaging. 2009; 53(1): 105-123, indexed in Pubmed: 19182734

38. Yuan K, Chen HL, Cui ZM. Diagnostic accuracy of C-reactive protein for periprosthetic joint infection: a meta-analysis. Surg Infect (Larchmt). 2014; 15(5): 548-559, doi: 10.1089/sur.2013.066, indexed in Pubmed: 24833084.

39. Glithero PR, Grigoris P, Harding LK, et al. White cell scans and infected joint replacements. Failure to detect chronic infection. J Bone Joint Surg Br. 1993; 75(3): 371-374, indexed in Pubmed: 8496202.

40. Claassen L, Radtke K, Ettinger M, et al. Preoperative diagnostic for periprosthetic joint infection prior to total knee revision arthroplasty. Orthop Rev (Pavia). 2014; 6(3): 5437, doi: 10.4081/or.2014.5437, indexed in Pubmed: 25317311.

41. Tam HH, Bhaludin B, Rahman F, et al. SPECT-CT in total hip arthroplasty. Clin Radiol. 2014; 69(1): 82-95, doi: 10.1016/j.crad.2013.08.003, indexed in Pubmed: 24047953.

42. Filippi L, Schillaci O. Usefulness of hybrid SPECT/CT in 99mTc-HMPAO-labeled leukocyte scintigraphy for bone and joint infections. J Nucl Med. 2006; 47(12): 1908-1913, indexed in Pubmed: 17138732.

43. Kim Hok, Na SJ, Oh SJ, et al. Usefulness of adding SPECT/CT to 99mTc-hexamethylpropylene amine oxime (HMPAO)-labeled leukocyte imaging for diagnosing prosthetic joint infections. J Comput Assist Tomogr. 2014; 38(2): 313-319, doi: 10.1097/RCT.0000000000000011, indexed in Pubmed: 24625603.

44. Graute V, Feist M, Lehner S, et al. Detection of low-grade prosthetic joint infections using 99mTc-antigranulocyte SPECT/CT: initial clinical results. Eur J Nucl Med Mol Imaging. 2010; 37(9): 1751-1759, doi: 10.1007/s00259010-1431-3, indexed in Pubmed: 20309680.

45. Gravius S, Gebhard M, Ackermann D, et al. [Analysis of 18F-FDG uptake pattern in PET for diagnosis of aseptic loosening versus prosthesis infection after total knee arthroplasty. A prospective pilot study]. Nuklearmedizin. 2010; 49(3): 115-123, doi: 10.3413/nukmed-0278, indexed in Pubmed: 20407734.

46. Basu S, Kwee TC, Saboury B, et al. FDG PET for diagnosing infection in hip and knee prostheses: prospective study in 221 prostheses and subgroup comparison with combined (111)In-labeled leukocyte/(99m)Tc-sulfur colloid bone marrow imaging in 88 prostheses. Clin Nucl Med. 2014; 39(7): 609-615, doi: 10.1097/RLU.0000000000000464, indexed in Pubmed: 24873788.

47. Zhuang $\mathrm{H}$, Duarte PS, Pourdehnad M, et al. The promising role of $18 \mathrm{~F}-\mathrm{FDG}$ PET in detecting infected lower limb prosthesis implants. J Nucl Med. 2001; 42(1): 44-48, indexed in Pubmed: 11197979

48. Cremerius $U$, Mumme T, Reinartz $P$, et al. [Analysis of (18)F-FDG uptake patterns in PET for diagnosis of septic and aseptic loosening after total hip arthroplasty]. Nuklearmedizin. 2003; 42(6): 234-239, indexed in Pubmed: 14668955.

49. Reinartz P, Mumme T, Hermanns B, et al. Radionuclide imaging of the painful hip arthroplasty: positron-emission tomography versus triple-phase bone scanning. J Bone Joint Surg Br. 2005; 87(4): 465-470, doi: 10.1302/0301-620X.87B4.14954, indexed in Pubmed: 15795194.

50. Stumpe KDM, Nötzli HP, Zanetti M, et al. FDG PET for differentiation of infection and aseptic loosening in total hip replacements: comparison with conventional radiography and three-phase bone scintigraphy. Radiology. 2004; 231(2): 333-341, doi: 10.1148/radiol.2312021596, indexed in Pubmed: 15044748

51. García-Barrecheguren E, Rodríguez Fraile M, Toledo Santana G, et al. [FDG-PET: a new diagnostic approach in hip prosthetic replacement]. Rev Esp Med Nucl. 2007; 26(4): 208-220, indexed in Pubmed: 17662187.

52. Stumpe KDM, Romero J, Ziegler O, et al. The value of FDG-PET in patients with painful total knee arthroplasty. Eur J Nucl Med Mol Imaging. 2006: 33(10): 1218-1225, doi: 10.1007/s00259-006-0127-1, indexed in Pubmed: 16721569 .

53. Delank KS, Schmidt M, Michael JWP, et al. The implications of 18F-FDG PET for the diagnosis of endoprosthetic loosening and infection in hip and knee arthroplasty: results from a prospective, blinded study. BMC Musculoskelet Disord. 2006; 7: 20, doi: 10.1186/1471-2474-7-20, indexed in Pubmed: 16512924

54. Jin H, Yuan L, Li C, et al. Diagnostic performance of FDG PET or PET/CT in prosthetic infection after arthroplasty: a meta-analysis. Q J Nucl Med Mol Imaging. 2014; 58(1): 85-93, indexed in Pubmed: 24469570. 